\title{
1 Ant collective behavior is heritable and shaped by selection
}

2

Justin T. Walsh ${ }^{*}$, Simon Garnier ${ }^{2}$, Timothy A. Linksvayer ${ }^{1}$

4

'Department of Biology, University of Pennsylvania, Philadelphia, PA 19104, USA

2Department of Biology, New Jersey Institute of Technology, Newark, NJ 07102, USA

$8 \quad *$ corresponding author, email: juswalsh@ sas.upenn.edu

\section{Abstract}

Collective behaviors are widespread in nature and usually assumed to be strongly shaped by natural selection. However, the degree to which variation in collective behavior is heritable

14 and has fitness consequences -- the two prerequisites for evolution by natural selection -- is largely unknown. We used a new pharaoh ant (Monomorium pharaonis) mapping population to

16 estimate the heritability, genetic correlations, and fitness consequences of three collective

17 behaviors (foraging, aggression, and exploration) as well as body size, sex ratio, and caste ratio.

18 Heritability estimates for the collective behaviors were moderate, ranging from 0.17 to 0.32 , but

19 lower than our estimates for the heritability of caste ratio, sex ratio, and the body size of new

20 workers, queens, and males. Moreover, variation among colonies in collective behaviors was

21 phenotypically correlated, suggesting that selection may shape multiple colony collective

22 behaviors simultaneously. Finally, we found evidence for directional selection that was similar in

23 strength to estimates of selection in natural populations. Altogether, our study begins to elucidate

24 the genetic architecture of collective behavior and is one of the first studies to demonstrate that it 25 is shaped by selection.

26 Keywords: Collective behavior, animal personality, genetic correlation, heritability, selection,

27 caste ratio 
bioRxiv preprint doi: https://doi.org/10.1101/567503; this version posted March 14, 2020. The copyright holder for this preprint (which was not certified by peer review) is the author/funder, who has granted bioRxiv a license to display the preprint in perpetuity. It is made available under aCC-BY-NC-ND 4.0 International license. 


\section{Introduction}

Collective behavior, defined as behaviors of groups of individuals that operate without central control (Gordon 2014, 2016), is ubiquitous in nature. Examples include predator avoidance in schools of fish, the migration of flocks of birds, and nest building in social insects. Increasingly, researchers have documented patterns of variation in collective behavior between groups (i.e. describing collective or group personality; Gordon 1991; Gordon et al. 2011; Jandt et evolutionary causes and consequences of variation in collective behavior. However, the degree to which collective behaviors are heritable and how genetic variation contributes to populationlevel variation in individual and collective behaviors remain largely unknown. Furthermore, it is often assumed that collective behavior and other group-level traits, like individual behavior and other individual-level traits, are strongly shaped by natural selection. However, little is actually

41 known about the fitness consequences of variation in collective behaviors, or group-level traits more generally (Gordon 2013, 2016; Wright et al. 2019).

Given that trait variation must be heritable in order for the trait to respond to selection

44 and evolve over time, quantifying heritability is a crucial first step in studying trait evolution

45 (Falconer \& Mackay 1996; Lynch \& Walsh 1998). Previous studies in ants, honey bees, and

46 sticklebacks suggest that collective behaviors and other group-level traits are heritable

47 (Linksvayer 2006; Hunt et al. 2007; Wark et al. 2011; Gordon 2013; Greenwood et al. 2015;

48 Friedman \& Gordon 2016). Additionally, candidate gene studies have linked allelic variation to

49 variation in collective behavior, providing further evidence that collective behavior is heritable

50 (Krieger 2005; Wang et al. 2008; Wang et al. 2013; Tang et al. 2018). Although numerous

51 studies have examined the genetic architecture of group-level traits in honey bees (Rinderer et al. 
52 1983; Collins et al. 1984; Milne 1985; Moritz et al. 1987; Bienefeld \& Pirchner 1990; Pirchner \&

53 Bienefeld 1991; Harris \& Harbo 1999; Boecking et al. 2000; Hunt et al. 2007), we know little

54 about the genetic architecture or the evolution of collective behavior and other group-level traits

55 in other group-living species.

Another key factor affecting the relationship between genotype, phenotype, and

57 evolutionary response to selection is the pattern of genetic correlations, i.e. the proportion of

58 variance that two traits share due to genetic causes. Genetic correlations can either accelerate or

59 slow down the rate of evolutionary response to selection, depending on the direction of the

60 correlation relative to the direction of selection on the traits (Lynch \& Walsh 1998; Wilson et al.

61 2010). Understanding genetic correlations is especially important for the study of behavioral

62 evolution since behaviors are often thought to be correlated with each other, forming sets of

63 tightly linked traits that are often described as behavioral syndromes (Sih et al. 2004;

64 Dochtermann \& Dingemanse 2013). Although genetic correlations have been estimated for

65 individual-level behaviors (reviewed by van Oers et al. 2005), few studies have examined

66 genetic correlations between collective behaviors or other group-level traits in social insects

67 (except for honey bees; Milne 1985; Bienefeld \& Pirchner 1990; Boecking et al. 2000).

The genetic architecture of group-level traits such as collective behavior is likely more

69 complex than the genetic architecture of individual-level traits, because variation in group-level

70 traits arises from phenotypic and genotypic variation within and among groups (Linksvayer

71 2006, 2015; Bijma et al. 2007a, 2007b; McGlothlin et al. 2010; Gempe et al. 2012). For example,

72 the genotype of each individual may influence its activity rate, which in turn may affect

73 interactions among group members and the collective performance of the group. Thus, group-

74 level traits depend on the genotypes of multiple interacting individuals, just as individual-level 
75 traits that are affected by social interactions, as considered in the interacting phenotypes

76 framework (Moore et al. 1997; McGlothlin et al. 2010). Indeed, previous honey bee studies

77 quantifying heritability for colony-level performance traits such as honey yield have treated

78 colony performance as a worker trait that is influenced by the expected genotype of workers and

79 also potentially influenced by the genotype of the queen (i.e. through a maternal genetic effect)

80 (Bienefeld and Pirchner 1990; Bienefeld and Pirchner 1991; Bienefeld et al. 2007; Brascamp et

81 al. 2016).

The rate and direction of a trait's potential evolutionary response to selection also

83 depends on the pattern of natural selection acting on the trait. Knowledge of the fitness

84 consequences of trait variation allows researchers to characterize the type (e.g., directional,

85 stabilizing, or disruptive) and strength of natural selection acting on a trait (Lande \& Arnold

86 1983; Arnold \& Wade 1984; Janzen \& Stern 1998; Morrissey \& Sakrejda 2013). Many studies

87 have estimated the fitness consequences of individual-level behavioral variation (reviewed by

88 Smith \& Blumstein 2008), but the consequences of group-level variation have received relatively

89 little attention (but see Wray et al. 2011; Modlmeier et al. 2012; Gordon 2013; Blight et al.

90 2016a; Blight et al. 2016b).

91 Social insects are well-established models for studying collective behavior. Well-studied

92 collective behaviors include nest choice in acorn ants (Temnothorax spp.; Möglich 1978; Franks

93 et al. 2003; Pratt 2017), nest defense and hygienic behavior in honey bees (Apis mellifera;

94 Spivak 1996; Breed et al. 2004; Evans \& Spivak 2010), and the regulation of foraging in pharaoh

95 ants (Monomorium pharaonis; e.g. Beekman et al. 2001; Sumpter \& Beekman 2003; Robinson et

96 al. 2005) and harvester ants (Pogonomyrmex barbatus; e.g. Gordon 2002; Greene \& Gordon

97 2007; Gordon et al. 2007; Gordon et al. 2011; Gordon 2013). The collective behavior of colony 
members also shapes colony productivity and the relative investment in workers versus reproductives (i.e. caste ratio) and reproductive males versus queens (i.e. sex ratio). Social insect sex ratio and caste ratio have long served as important models for empirically testing predictions

101 from inclusive fitness theory regarding predicted conflicts between queens and workers over sex 102 ratio and caste ratio (Trivers \& Hare 1976; Reuter \& Keller 2001; Mehdiabadi et al. 2003;

103 Linksvayer 2008; Bourke 2015;). However, despite this long-term intense interest in the 104 evolution of colony-level traits, empirical evidence is scarce about the key parameters governing 105 the evolution of these traits, especially for ants. Indeed, while recent molecular studies have 106 begun to characterize the genomic, transcriptomic, and epigenetic differences between species, 107 between castes within a species, and between individual workers (Friedman \& Gordon 2016;

108 Gospocic et al. 2017; Warner et al. 2017; Chandra et al. 2018; Walsh et al. 2018), little is known 109 about the genetic architecture of collective behavior, caste ratio, and sex ratio (Linksvayer 2006). 110 Similarly, while it is clear that colony-level phenotypes can be shaped by patterns of selection 111 within- and between-colonies (Owen 1986; Moritz 1989; Ratnieks \& Reeve 1992; Tsuji 1994,

112 1995; Banschbach \& Herbers 1996; Tarpy et al. 2004; Gordon 2013), few studies have attempted 113 to empirically quantify patterns of selection acting on social insect traits. In this study we used a genetically and phenotypically variable laboratory population of

115 pharaoh ants (Monomorium pharaonis). Such a mapping population has proven powerful to 116 elucidate the genetic architecture of a range of traits, including behavioral traits, in mice, rats, 117 and fruit flies (Hansen \& Spuhler 1984; Mott et al. 2000; Valdar et al. 2006; King et al. 2012).

118 We first assayed colony-level foraging, aggression, and three measures of exploration using three 119 replicate sub-colonies of 81 distinct colony genotypes of known pedigree (243 replicate sub120 colonies total). Collective behaviors are defined as emergent behaviors of groups of individuals 
121 that operate without central control, through local interactions (Gordon 2014, 2016). We

122 consider the behaviors of foraging, exploration, and aggression to be collective because all three

123 consist of emergent patterns of workers operating at least in part through local interactions, either

124 through direct antennal contact with other workers or through the influence of pheromones

125 (Adler \& Gordon 1992; Gordon \& Mehdiabadi 1999; Gordon 2002, 2010; Greene \& Gordon

126 2007; Pinter-Wollman et al. 2013; Kleineidam et al. 2017). In many social insects, foragers are

127 stimulated to begin foraging through interactions with other foragers/scouts returning to the nest

128 (e.g. Gordon 2002; Fernandez et al. 2003; Pinter-Wollman et al. 2013). Both foraging and

129 exploratory behavior are often regulated through the use of trail pheromones (e.g. Fourcassie \&

130 Deneubourg 1994; Jackson \& Châline 2007). During aggressive responses to threats, workers are

131 often recruited via the use of alarm pheromones (Loftqvist 1976; Blum 1996) or through social

132 interactions with other workers (Kleineidam et al. 2017). We also chose these collective

133 behaviors because they are linked to colony success in other social insects, including other

134 species of ants (Wray et al. 2011; Modlmeier et al. 2012; Blight et al. 2016a; Blight et al. 2016b).

135 Furthermore, we measured colony productivity, caste and sex ratio, and worker, gyne, and male

136 body size. We used the known pedigree of colonies in our mapping population, together with

137 trait measurements in an animal model framework, to estimate the heritability of and genetic

138 correlations between all traits. Finally, we estimated the strength and pattern of selection acting

139 on all the measured phenotypes in the laboratory.

141 Materials and Methods

142 (a) Background and overall design 
All M. pharaonis colonies used in this study were reared in the lab and derived from

144 eight initial lab stocks, collected from eight different locations across Africa, Asia, Europe, and

145 North America (Schmidt 2010; Schmidt et al. 2010). Specifically, the eight initial stocks were

146 systematically intercrossed for nine generations in order to create a mapping population that was

147 initially designed to be analogous to the mouse heterogeneous stock (Mott et al. 2000; Valdar et

148 al. 2006). After nine generations of intercrossing, each colony in the resulting mapping

149 population is expected to contain a unique mixture of alleles from the eight initial stocks

150 (Pontieri et al. 2017) (Supplemental figure 1). We maintained all colonies at $27 \pm 1{ }^{\circ} \mathrm{C}$ and $50 \%$

151 relative humidity on a 12:12 hour light:dark cycle. We split each colony (henceforth "colony

152 genotype") into three equally-sized replicates (henceforth "colony replicate") by emptying the

153 colony genotypes into plastic bowls, gently mixing the queens, workers, and brood, and using tea

154 spoons to scoop them into three new colony containers. Next, we manually counted all

155 individuals within the colony replicates and adjusted the numbers accordingly so that all colony

156 replicates initially consisted of 4 queens, $400 \pm 40$ workers, $60 \pm 6$ eggs, $50 \pm 5$ first instar larvae,

$15720 \pm 2$ second instar larvae, $70 \pm 7$ third instar larvae, $20 \pm 2$ prepupae, and $60 \pm 6$ worker pupae.

158 These numbers represent a typical distribution of developmental stages in a relatively small $M$.

159 pharaonis colony (Warner et al. 2018). Except when starving the colony replicates (see below),

160 we fed all colony replicates twice per week with an agar-based synthetic diet (Dussutour \&

161 Simpson 2008) and dried mealworms. The colony replicates always had access to water via

162 water tubes plugged with cotton and nested between two glass slides $(5 \mathrm{~cm} \mathrm{x} 10 \mathrm{~cm})$. We kept all

163 colony replicates in a plastic colony container $(18.5 \mathrm{~cm}$ x $10.5 \mathrm{~cm} \times 10.5 \mathrm{~cm})$ lined with fluon

164 and surrounded by a moat of oil to prevent the workers from escaping the box. 
After setting up the colony replicates, we gave them two weeks to acclimate to the new

166 conditions before conducting behavioral assays. We fed the colony replicates twice per week

167 except for the week prior to the exploratory and foraging assays during which we starved the

168 colony replicates so that they would be motivated to explore and forage. We conducted the

169 exploratory and foraging assays during the third week and the aggression assays during the

170 fourth week after setting up the replicate colonies.

171 (b) Behavioral observations

172 (i) Exploratory assay

173 We conducted the exploratory assay after the colony replicates had been starved for six

174 days. We assayed the exploratory behavior of both entire colony replicates and groups of five

175 foragers. We conducted the assay inside a filming box with white LED lights arranged along the

176 walls and a camera mounted on the top to film the arena from above (Supplemental figure 2A).

177 To remove trail pheromones between assays, we covered the floor of the box with white poster

178 board that we replaced between each assay. We first collected five foragers, defined as any

179 worker outside the nest, from inside the colony container and placed them in a large petri dish.

180 We placed the petri dish upside-down in the middle of a circular arena in the center of the

181 filming box and waited five minutes to give the workers time to settle down after being handled.

182 After the five minutes, we gently removed the petri dish so the workers were free to move

183 around the arena and filmed the workers exploring the arena for 15 minutes.

Next, we replaced the poster board inside the filming box and placed the five foragers, all

185 remaining foragers from inside the colony container, and the nest containing the rest of the

186 workers, queens, and brood inside a petri dish. We placed the petri dish containing the entire 
colony upside-down in the center of the arena and waited five minutes before lifting the petri

188 dish and filming for 15 minutes.

We analyzed the videos of the five foragers using custom made tracking software

190 (https://github.com/swarm-lab/trackR; accessed 2017) to track the location of each ant in each

191 frame of the video. To avoid the effect of the arena wall on ant trajectories, we removed all

192 tracks where the ants were within $3 \mathrm{~mm}$ of the wall, resulting in many separate trajectories

193 within each video for each ant. Next, for each sub-trajectory, we calculated the net squared

194 displacement (NSD) by taking the square of the distance traveled by each ant between the

195 starting location and each successive location along the rest of the trajectory. To calculate the

196 diffusion coefficient, we took the slope of the plot of NSD over time and fit the equation:

$$
M S D=4 D t
$$

where mean squared displacement $(M S D)$ is the slope of NSD over time, $D$ is the diffusion coefficient, and $t$ is time (Börger \& Fryxell 2012). The diffusion coefficient served as a measure of how quickly the ants collectively explored a novel space.

204 without ants in it. When a pixel had a large absolute difference, it meant an ant was present on 205 that pixel in a given frame. We then applied a threshold to the difference image and classified all 206 the pixels with a difference value above the threshold as "ant-covered" pixels and gave them a 207 value of 1 , and all the pixels with a difference value below the threshold as "background" pixels 208 and gave them a value of 0 . Finally, we computed the cumulative sum of the segmented images 209 over time and calculated for each of them the arena coverage as the percentage of the pixels with 
210 a value of at least 1 (i.e. what fraction of pixels have been visited by ants at least once; Figure

211 1).

212

We will refer to three exploratory behaviors as "exploratory rate", "group exploration",

213 and "colony exploration". "Exploratory rate" refers to the diffusion coefficient of groups of five

214 ants, "group exploration" to the percent of the arena covered by the groups of five foragers, and

215 "colony exploration" to the percent of the arena covered by the entire colony.

\section{6 (ii) Foraging assay}

We conducted the foraging assay on each colony replicate the day after the exploratory

218 assay and after the colony replicates had been starved for a week. We melted the agar-based

219 synthetic diet and soaked a cotton ball in the liquid. When the cotton ball solidified, we placed it

220 on the plateau of a 3D printed ramp and placed the ramp inside a colony container on the

221 opposite site of the nest (Supplemental figure 2B). Once an ant first discovered the food, we

222 started filming and filmed for one hour. If no ant discovered the food in 30 minutes, we started

223 the recording. We calculated the foraging rate by manually counting the number of ant visits to

224 the plateau of the ramp in each video. Because many ants went back and forth from the food to

225 the nest, we counted many ants more than once.

\section{6 (iii) Aggression assay}

Like other unicolonial ant species, M. pharaonis workers show little to no aggression

229 workers to act aggressively, and to be able to quantify aggression against a constant "enemy" for

230 all of our experimental colonies, we used workers from a single Monomorium dichroum colony

231 that had been kept in the lab under the same conditions as the M. pharaonis colonies for 5 years.

232 We conducted the aggression assays a week after the foraging assays. We first collected twenty 
233 foragers of both species and placed them in separate small petri dishes (Supplemental figure 3).

234 We placed both small petri dishes upside down in a large petri dish for five minutes before lifting

235 both petri dishes and allowing the workers of both species to interact. Every 5 minutes for one

236 hour, we manually counted and recorded the number of $M$. pharaonis workers that were biting

237 M. dichroum workers. We defined aggression as the average number of M. pharaonis workers

238 biting $M$. dichroum workers across all observations within an hour. We froze all of the ants used

239 in the aggression assay so that we did not reuse $M$. dichroum workers in more than one assay.

240 (c) Colony productivity and body mass measurements

241 As a measure of colony productivity, we surveyed each colony replicate once per week

242 and counted the number of workers and brood at all developmental stages. $M$. pharonis colonies

243 usually only produce new gynes (virgin queens) and males in the absence of fertile queens

244 (Edwards 1991; Warner et al. 2018). Therefore, in order to induce the production of new gynes

245 and males, we removed queens at the start of the fifth week, after the aggression assay. We

246 conducted weekly surveys until all brood matured into worker, gyne, or male pupae. In addition

247 to colony productivity data for the total number of workers, gynes, and males produced, the

248 weekly surveys also allowed us to calculate colony caste and sex ratio. We defined caste ratio as

249 the number of gynes relative to the total number of females produced, and sex ratio as the

250 number of gynes relative to the total number of reproductives (gynes and males) produced. To

251 measure body size, we collected 15 worker pupae, 10 gyne pupae, and 10 male pupae from each

252 colony replicate. We dried the pupae out in a drying oven for 24 hours before weighing.

\section{3 (d) Heritability and genetic correlation analysis}

254 We performed all statistical analyses in $\mathrm{R}$ version 3.4.1 (R Core Team 2014). We

255 estimated the repeatability of all measured phenotypes across colony replicates using a 
generalized linear mixed model (GLMM) approach in the R package MCMCglmm (Hadfield

257

258

259

260

261

262

263

264

265

266

267

268

269

270

271

272

273

274

275

276

277

278

2010). We included block as a random factor to account for the fact that the samples were

collected at different time points from the replicate colonies and included colony identity as a

random effect and Wolbachia infection status as a fixed effect (two of the original eight lineages

included in the heterogeneous stock were infected with Wolbachia; Schmidt et al. 2010, Pontieri

et al. 2017).

To estimate the heritability of, and genetic correlations between, all measured

phenotypes, we used an animal model approach. Animal models estimate genetic parameters of

traits by evaluating how patterns of observed phenotypic covariance between all pairs of

individual "animals" is predicted by the expected genetic relatedness between individuals, based

on pedigree (Kruuk 2004, de Villemereuil 2012). For our study, “individual animals" were

replicate colonies, the pedigree was the known pedigree across nine generations of the $M$.

pharaonis colonies in our mapping population, and the pedigree specifically represented

genealogical relationships among the workers (i.e. the worker offspring of queen and male

parents) that make up the replicate colonies of the mapping population. We thus assessed the

degree to which the expected genotype of workers predicted the observed collective behavior or

group-level phenotype measured for groups of workers from replicate colonies. Note that while

we focused only on how expected worker genotype was associated with variation in worker

collective behavior and colony-level traits, it is certainly possible that the genotypes of other

types of colony members (i.e. queens or sibling larvae) also contributes to variation in the group-

level traits we measured. Such effects can be independently estimated as described above, if very

large datasets are available (e.g., Brascamp et al. 2016 used a honey bee dataset with 15,000

colonies), or alternatively, these effects can be experimentally teased apart with cross-fostering 
(Linksvayer 2006, 2007, Linksvayer et al. 2009). However, we did not have enough power in our dataset to separately estimate potential queen genetic effects, and effects of larval genotype are always completely confounded with worker genotype barring experimental cross-fostering. Specifically, we used the R package MCMCglmm to run animal models using a Bayesian Markov chain Monte Carlo (MCMC) approach (de Villemereuil 2012). We accounted for the

284 fact that ants are haplodiploid (males are haploid, females are diploid) by constructing the 285 pedigree as if the traits were all sex-linked (Hedrick \& Parker 1997). We used weakly 286 informative priors for 1,000,000 iterations, with a burn-in period of 10,000 iterations and stored 287 estimates every 500 iterations (full R script included in supplemental material; following de Villemereuil 2012). We assessed convergence of the models by visually inspecting estimate plots and assessing the autocorrelation values (de Villemereuil 2012). We analyzed whether behaviors were phenotypically correlated with each other (i.e. behavioral syndromes) using Spearman rank

291 correlations and corrected for multiple comparisons by using the "FDR" method in the R 292 function "p.adjust." In our initial heritability estimates, we ignored two complications in our pedigree. First,

294 between our conducting new crosses to produce new generations, our colonies went through 295 multiple rounds of intranidal mating: when the fecundity of current queens declines, $M$.

296 pharaonis colonies produce new gynes and males which stay in the nest and mate with each 297 other (Berndt \& Eichler 1987). Second, when a colony was the mother/father colony to multiple 298 offspring colonies, we initially treated those offspring colonies as half siblings. However, 299 because $M$. pharaonis colonies contain multiple queens, the new gynes and males they produce may be better thought of as cousins. To test whether either of these complications would affect 301 our heritability estimates, we constructed multiple pedigrees and re-ran the heritability analyses. 
302 We constructed pedigrees in which one or two generations contained two rounds of intranidal

303 mating, one or two generations considered reproductives from the same colony as cousins, and

304 two generations of both intranidal mating and considering reproductives from the same colony as

305 cousins.

(e) Selection analysis

We defined fitness in two ways, as either the production of new reproductives (gynes or males) or new workers, and ran separate models for each fitness definition. In nature, $M$. pharaonis colonies reproduce by budding (i.e. new colonies are not founded independently by

310 queens; Buczkowski \& Bennett 2009), but instead, a number of queens and workers disperse

311 with brood to form a new colony. Both new reproductives and new workers determine the

312 growth rate and potential to bud for existing colonies, and hence are appropriate measures of

313 colony fitness. We estimated the strength of selection using a multivariate standardized selection

314 gradient approach as described by Morrissey \& Sakrejda (2013). This method is similar to the

315 approach outlined by Lande and Arnold (1983) and uses spline-based generalized additive

316 models to model the relationship between fitness and traits. We normalized all behaviors to a

317 mean of zero and a standard deviation of one so that the selection estimates represent

318 standardized values (Lande \& Arnold 2983; Morrissey \& Sakrejda 2013). We included all five

319 behaviors and block in all models and estimated selection gradients and prediction intervals after

3201000 bootstrap replicates (Morrissey \& Sakrejda 2013).

\section{Results}

323 (a) Repeatability and heritability estimates 
All five behaviors, caste and sex ratio, and worker, gyne, and male body mass were

325

326

327

328

329

330

331

332

333

334

335

336

337

338

339

340

341

342

343

344

345

346

significantly repeatable across replicate colonies (Supplemental table 1). We estimated the heritability of the five collective behaviors to be between 0.17 and 0.32 , with a median value of 0.21 (Figure 2). We estimated the heritability of worker body mass to be 0.34 , gyne body mass to be 0.46 , and male body mass to be 0.53 (Figure 2). We estimated the heritability of five colony productivity measures to be between 0.001 and 0.46 , with a median value of 0.24 (Figure 2). Finally, we estimated the heritabilty of colony caste and sex ratio to be 0.26 and 0.23 , respectively (Figure 2).

We compared our initial heritability estimates with heritability estimates using five different modified pedigrees that considered intranidal mating and/or considering offspring colonies as cousins rather than half siblings. The difference between the initial heritability estimates and the estimates when using the five modified pedigrees were small, less than 0.1 for all phenotypes except sex ratio, which differed by up to 0.24 (Supplemental table 2).

\section{(b) Phenotypic and genetic correlation estimates}

We found phenotypic correlations among the five measured collective behaviors (Figure

3). Foraging rate was negatively correlated with aggression and positively correlated with both

group exploration and colony exploration. Aggression was negatively correlated with

exploratory rate. Group exploration and colony exploration were positively correlated. The genetic correlation estimates ranged from -0.05 to 0.17 but the $95 \%$ CIs all overlapped with zero (see Figure 3 and Supplemental table 3 for estimates and 95\% CI). The genetic correlation estimates between behaviors and all other traits, as well as among all the other traits, were mostly small and all had 95\% CI that overlapped with zero (Supplemental table 4). 
(c) Selection gradients

When defining fitness as the number of reproductives (gynes + males) produced by the

350 colony, we found evidence for positive linear selection on foraging and negative linear selection

351 on exploratory rate (Table 1, Figure 4). We found no evidence for quadratic selection. When

352 defining fitness as the number of workers produced by a colony, we found evidence for positive

353 linear selection on foraging and no evidence for quadratic selection (Table 1, Figure 4). When

354 defining fitness as either the production of new reproductives or workers, we found no evidence

355 for correlational selection between any of the five behaviors. Finally, we found no evidence for

356 linear or quadratic selection on worker, gyne, or male body mass (Supplemental table 5).

357 To further put our results into context, we estimated the proportion of variance among

358 our colonies for both measures of fitness (the productions of new reproductives and workers) that

359 was explained by variation in any of our five behavioral variables, experimental block, or

360 Wolbachia infection status. For the production of new reproductives, we found that aggression

361 explained the largest amount of the variance (5.29\%), followed by foraging (2.29\%), group

362 exploration (1.94\%), exploratory rate (0.52\%), and colony exploration $(0.33 \%)$ (Supplemental

363 table 6). For the production of new workers, we found that foraging explained the largest

364 amount of the variance (1.29\%), followed by aggression (0.53\%), colony exploration $(0.34 \%)$,

365 group exploration (0.27\%), and exploratory rate (0.08\%) (Supplemental table 6).

367 Discussion

Collective behavior is ubiquitous in nature and presumed to have strong fitness

369 consequences for group members. Moreover, repeatable variation in collective behavior (often 
370 described as collective or group-level "personality") has been commonly observed (Bengston \&

371 Jandt 2014; Planas-Sitjà et al. 2015; Jolles et al. 2017; Wright et al. 2019). However, little is

372 known about the heritability or genetic architecture of collective behavior and how collective

373 behavior is shaped by selection. A major difficulty for elucidating the genetic basis of collective

374 behavior is that, unlike individual-level behavior, collective behavior by definition depends on

375 social interactions among members of the group. As a result, the genetic architecture of

376 collective behavior fundamentally depends on the collective genetic make-up of these

377 individuals (McGlothlin et al. 2010; Linksvayer 2006, 2015). Quantifying patterns of selection

378 on group-level traits also has an added level of difficulty because the level of replication is the

379 group (e.g., colony) and not the individual. Here, we begin to elucidate the genetic architecture

380 underlying collective behavior and other group-level traits and to characterize how selection acts

381 on these traits in a laboratory population of the ant Monomorium pharaonis that we created for

382 this purpose. We provide evidence that variation in collective behaviors, including foraging,

383 aggression, exploratory rate, group and colony exploration, and other group-level traits measured

384 in the laboratory is heritable, phenotypically and genetically correlated, and shaped by selection.

385 We estimated the heritability of collective behaviors to be between 0.22 and 0.40 , which

386 was generally lower than the heritability estimates for body size ( 0.38 to 0.58$)$, colony

387 productivity (0.14 to 0.75$)$, and caste (0.42) and sex ratio (0.49) (Figure 2, also see

388 Supplemental table 2 for heritability estimates using more complex pedigrees). These

389 heritability estimates demonstrate that all of the phenotypes we measured, including collective

390 behaviors, have the ability to respond to short term selection on standing genetic variation.

391 Although numerous studies have examined the genetic architecture of group-level traits in honey

392 bees (Rinderer et al. 1983; Collins et al. 1984; Milne 1985; Moritz et al. 1987; Bienefeld \& 

al. 2007), this is one of the first studies to examine the genetic architecture or the evolution of collective behavior and other group-level traits in an ant species.

Although our heritability estimates are somewhat higher than other estimates of

397 heritability across animal taxa (e.g. the heritability of individual-level behaviors was on average expected to be higher in animals bred in captivity than in nature because environmental conditions in the laboratory are controlled (Simmons \& Roff 1994). Furthermore, the heritability

401 estimates for all of our measured group-level phenotypes may be higher than individual-level

402 behaviors because the heritability of traits influenced by social interactions includes the 403 contribution of heritable components of the social environment (Linksvayer 2006; Bijma et al.

404 2007a, 2007b; Linksvayer et al. 2009; McGlothlin et al. 2010; Bijma 2011). There is ample

405 empirical and theoretical evidence that this form of "hidden heritability" contributes to the 406 heritable variation and also the evolutionary response to selection for social traits (Wade 1976;

407 Moore 1990; Muir 2005; Linksvayer 2006; Bijma et al. 2007b; Bergsma et al. 2008; Wade et al.

408 2010; Bijma 2011). Because we kept all components of the social environment intact across

409 replicate sub-colonies of each colony genotype (i.e. the workers, queens and brood were all from

410 the same parent colony), our heritability estimates do not partition out the relative contributions

411 of variation in the workers' own genomes from variation in the genomes of other colony

412 members (Linksvayer 2006; Linksvayer et al. 2009).

413 We found evidence for both phenotypic and genetic correlations between collective

414 behaviors. Suites of phenotypically correlated behaviors are termed "behavioral syndromes" and 415 have been documented throughout the animal kingdom, including in social insects (Sih et al. 
2004; Jandt et al. 2014). The behavioral syndrome we found in M. pharaonis consisted of a

417 positive correlation between foraging and exploration, which were both negatively correlated

418 with aggression. Our phenotypic and genetic correlation estimates were generally similar. For

419 example, the four strongest genetic correlation estimates (Foraging - Aggression; Foraging -

420 Forager coverage; Foraging - Colony coverage; Forager coverage - Colony coverage; Figure 3)

421 were also four of the five significant phenotypic correlations and were all in the same direction.

422 However, our genetic correlation estimates were generally very weak (i.e. not significantly

423 different than zero) and only one of our genetic correlation estimates was bound away from zero

424 (the correlation between foraging and colony exploration).

426 were heritable, not strongly genetically correlated, and thus free to evolve independently from

427 other traits in response to patterns of selection on each trait. This approach was termed the

428 "phenotypic gambit" (Grafen 1984). Our results generally support these assumptions as we

429 found moderate estimates of heritability for all five behavioral variables and relatively weak

430 genetic correlation estimates. These results suggest that collective behaviors are free to respond

431 to selection, and that the underlying genetic architecture will not constrain long-term

432 optimization by natural selection (Lynch \& Walsh 1998; Wilson et al. 2010).

433 We calculated the strength and direction of selection acting on collective behavior and

434 found evidence for both positive and negative linear selection (Figure 4, Table 1). The absolute

435 value of our estimates of the strength of linear and quadratic selection are similar or slightly

436 smaller than estimates of selection in wild populations (Kingsolver et al. 2001). The strongest

437 pattern of linear selection we found was for foraging, indicating that colonies with higher

438 foraging rates produced more reproductives as well as workers. A higher foraging rate is 
439 presumably associated with higher input of resources for the colony, allowing colonies to be

440 more productive.

441 We conducted the current study in a laboratory environment, which enabled us to strictly

442 control the demographic make-up (i.e. queen number, worker number, etc.) and precise

443 environmental conditions experienced by the three colony replicates for each of our 81 colony

444 genotypes. Such control in particular is valuable given the complexity of social insect colonies

445 (Linksvayer 2006; Kronauer \& Libbrecht 2018). However, we also acknowledge the caveat that

446 our choice to conduct our study in a controlled laboratory environment likely had strong effects

447 on both our estimates of heritability and genetic correlations, as well as our estimates of the

448 pattern and magnitude of selection. In particular, it is difficult to know how the fitness

449 consequences of variation in collective behavior that we observed would change in a more

450 natural setting. One possibility is that we might observe positive linear selection for aggression

451 since aggression has no obvious benefit in the lab but may have benefits in nature. Laboratory-

452 based estimates of natural selection are commonly used to test predictions of evolutionary theory

453 (Fuller et al. 2005). For example, researchers used lab-based manipulations to test predictions of

454 density-dependent selection theory in Drosophila melanogaster (e.g. Mueller 1997; Dasgupta et

455 al. 2019). Our study provides some of the first evidence that natural selection can shape

456 collective phenotypes, an assumption that is rarely tested, on a scale that is likely not feasible in

457 a field study. Furthermore, because $M$. pharaonis tends to be found in association with humans,

458 both in the tropics in their presumed native range (Wetterer 2010) and in heated buildings in

459 introduced temperate regions, the laboratory conditions of our study might be more similar to the

460 "natural" conditions experienced by our study species than other non-synanthropic species. 
Overall, this study increases our understanding of the genetic architecture of collective

462 behavior and demonstrates that it is strongly shaped by natural selection. Future studies should

463 focus on identifying the mechanisms by which genes function to influence collective behavior

464 and how variation in these genes affects patterns of variation for collective behavior within

465 populations. Candidate gene approaches have been used successfully to demonstrate the roles of

466 the ant ortholog of the foraging gene (Ingram et al. 2005; Lucas \& Sokolowski 2009; Ingram et

467 al. 2016; Bockoven et al. 2017; Page et al. 2018) and dopamine (Friedman et al. 2018). In

468 addition to candidate gene approaches, future studies should utilize unbiased approaches such as

469 quantitative trait locus (QTL) mapping in mapping populations (e.g., Hunt et al. 1998; 2007)

470 such as ours, and association mapping in natural populations (e.g., Kocher et al. 2018).

471 Additionally, future research should aim to understand the mechanisms underlying the

472 expression of collective behavior (Friedman et al. 2019). For example, chemical communication

473 (e.g. cuticular hydrocarbons, pheromones) likely plays a large role in regulating collective

474 behavior in social insects. Finally, future studies should seek to disentangle the contribution of

475 workers' own genomes and the composite sociogenome of their nestmates (including other

476 workers, queens, and brood), by using cross-fostering approaches and experimentally setting up

477 mixed worker groups (Morowitz \& Southwick 1987; Calderone \& Page 1992; Linksvayer 2006;

478 Linksvayer et al. 2009; Gempe et al. 2012).

479

480 Competing Interests

$481 \quad$ We have no competing interests. 
484

485

486

487

488

489

490

491

492

493

494

495

496

497

498

499

500

501

502

503

504

505

Adler, F. R., \& Gordon, D. M. (1992). Information collection and spread by networks of patrolling ants. The American Naturalist, 140(3), 373-400.

Arnold, S. J. \& Wade, M. J. 1984. On the measurement of natural and sexual selection: theory. Evolution 38: 709-719.

Banschbach, V. S. \& Herbers, J. M. 1996. Complex colony structure in social insects: II. Reproduction, queen-worker conflict, and levels of selection. Evolution 50: 298-307.

Beekman, M., Sumpter, D. J. T. \& Ratnieks, F. L. W. 2001. Phase transition between disordered and ordered foraging in Pharaoh's ants. Proceedings of the National Academy of Sciences 98: 9703-9706.

Bengston, S. E. \& Jandt, J. M. 2014b. The development of collective personality: the ontogenetic drivers of behavioral variation across groups. Frontiers in Ecology and Evolution 2.

Bergsma, R., Kanis, E., Knol, E. F. \& Bijma, P. 2008. The contribution of social effects to heritable variation in finishing traits of domestic pigs (Sus scrofa). Genetics 178: 15591570.

Berndt, K.-P. \& Eichler, W. 1987. Die Pharaoameise, Monomorium pharaonis (L.) (Hym., Myrmicidae). Mitteilungen aus dem Museum für Naturkunde in Berlin. Zoologisches Museum und Institut für Spezielle Zoologie (Berlin) 63: 3-186.

Bienefeld, K., Ehrhardt, K. \& Reinhardt, F. 2007. Genetic evaluation in the honey bee considering queen and worker effects-A BLUP-Animal Model approach. Apidologie 38: 77-85.

Bienefeld, K. \& Pirchner, F. 1990. Heritabilities for several colony traits in the honey bee (Apis mellifera carnica). Apidologie 21: 175-183. 
Bijma, P. 2011. A general definition of the heritable variation that determines the potential of a population to respond to selection. Genetics 189: 1347-1359. selection 2: Estimating the genetic parameters determining inheritance and response to

511 Bijma, P., Muir, W. M. \& Van Arendonk, J. A. M. 2007b. Multilevel selection 1: Quantitative genetics of inheritance and response to selection. Genetics 175: 277.

Blight, O., Albet Díaz-Mariblanca, G., Cerdá, X. \& Boulay, R. 2016a. A proactive-reactive syndrome affects group success in an ant species. Behavioral Ecology 27: 118-125.

Blight, O., Villalta, I., Cerdá, X. \& Boulay, R. 2016b. Personality traits are associated with colony productivity in the gypsy ant Aphaenogaster senilis. Behavioral Ecology and

Blum, M. S. (1996). Semiochemical parsimony in the Arthropoda. Annual Review of Sociobiology 70: 2203-2209.

Bockoven, A. A., Coates, C. J. \& Eubanks, M. D. 2017. Colony-level behavioural variation correlates with differences in expression of the foraging gene in red imported fire ants.

523 Boecking, O., Bienefeld, K. \& Drescher, W. 2000. Heritability of the Varroa-specific hygienic behaviour in honey bees (Hymenoptera: Apidae). Journal of Animal Breeding and Genetics 117: 417-424.

526 Börger, L. \& Fryxell, J. (2012) Quantifying individual differences in dispersal using net squared displacement. pp. 222-230. 
Bourke, A. F. G. 2015. Sex investment ratios in eusocial Hymenoptera support inclusive fitness theory. Journal of Evolutionary Biology 28: 2106-2111. and genetic correlations for honey yield, gentleness, calmness and swarming behaviour in

Breed, M. D., Diaz, P. H. \& Lucero, K. D. 2004. Olfactory information processing in honeybee, Apis mellifera, nestmate recognition. Animal Behaviour 68: 921-928.

Buczkowski, G., \& Bennett, G. (2009). Colony budding and its effects on food allocation in the highly polygynous ant, Monomorium pharaonis. Ethology, 115(11), 1091-1099.

537 Calderone, N. W. \& Page, R. E. 1992. Effects of interactions among genotypically diverse nestmates on task specialization by foraging honey bees (Apis mellifera). Behavioral

540 Chandra, V., Fetter-Pruneda, I., Oxley, P. R., Ritger, A. L., McKenzie, S. K., Libbrecht, R. \& Kronauer, D. J. C. 2018. Social regulation of insulin signaling and the evolution of

543 Collins, A. M., Rinderer, T. E., Harbo, J. R. \& Brown, M. A. 1984. Heritabilities and correlations for several characters in the honey bee. Journal of Heredity 75: 135-140.

545 Dasgupta, P., Sarkar, S., Das, A. A., Verma, T., \& Nandy, B. (2019). Intergenerational paternal effect of adult density in Drosophila melanogaster. Ecology and Evolution, 9(6), 3553-

548 de Villemereuil, P. 2012. Tutorial estimation of a biological trait heritability using the animal model: How to use the MCMCglmm R package. 
Dochtermann, N. A. \& Dingemanse, N. J. 2013. Behavioral syndromes as evolutionary constraints. Behavioral Ecology 24: 806-811.

Dochtermann, N. A., Schwab, T. \& Sih, A. 2015. The contribution of additive genetic variation to personality variation: heritability of personality. Proc Biol Sci 282: 20142201.

Dussutour, A. \& Simpson, S. J. 2008. Description of a simple synthetic diet for studying nutritional responses in ants. Insectes Sociaux 55: 329-333.

Edwards, J. P. 1991. Caste regulation in the pharaohs ant Monomorium pharaonis- recognition and cannibalism of sexual brood by workers. Physiological Entomology 16: 263-271.

558 Evans, J. D. \& Spivak, M. 2010. Socialized medicine: Individual and communal disease barriers in honey bees. Journal of Invertebrate Pathology 103: S62-S72.

560 Falconer, D. S. \& Mackay, T. F. C. 1996. Introduction to quantitative genetics, 4th ed. Prentice Hall, Esses.

562 Fernández, P. C., Gil, M., \& Farina, W. M. (2003). Reward rate and forager activation in honeybees: recruiting mechanisms and temporal distribution of arrivals. Behavioral

565 Fourcassie, V., \& Deneubourg, J. L. (1994). The dynamics of collective exploration and trailformation in Monomorium pharaonis: experiments and model. Physiological Entomology, 19(4), 291-300. collective decision making. Proceedings of the Royal Society of London. Series B:

571 Friedman, D. A. \& Gordon, D. M. 2016. Ant genetics: reproductive physiology, worker morphology, and behavior. Annual Review of Neuroscience 39: 41-56. 
573 Friedman, D. A., Pilko, A., Skowronska-Krawczyk, D., Krasinska, K., Parker, J. W., Hirsh, J. \&

574 Gordon, D. M. 2018. The role of dopamine in the collective regulation of foraging in

$575 \quad$ harvester ants. iScience 8: 283-294.

576 Friedman, D. A., Johnson, B. R., \& Linksvayer, T. A. (2019). Decentralized physiology and the 577 molecular basis of social life in eusocial insects. arXiv, 1911.01321.

578 Fuller, R. C., Baer, C. F., \& Travis, J. (2005). How and when selection experiments might actually be useful. Integrative and Comparative Biology, 45(3), 391-404.

580 Gempe, T., Stach, S., Bienefeld, K. \& Beye, M. 2012. Mixing of honeybees with different genotypes affects individual worker behavior and transcription of genes in the neuronal substrate. PLOS ONE 7: e31653. American Naturalist 138: 379-411.

Gordon, D. M. (2010). Ant encounters: interaction networks and colony behavior (Vol. 1): Princeton University Press.

587 Gordon, D. M. 2013. The rewards of restraint in the collective regulation of foraging by $588 \quad$ harvester ant colonies. Nature 498: 91.

589 Gordon, D. M. 2014. The ecology of collective behavior. PLOS Biology 12: e1001805.

590 Gordon, D. M. 2016. The evolution of the algorithms for collective behavior. Cell Systems 3: 514-520.

592 Gordon, D. M. 2002. The regulation of foraging activity in red harvester ant colonies. The 593 American Naturalist 159: 509-518.

594 Gordon, D. M., Guetz, A., Greene, M. J. \& Holmes, S. 2011. Colony variation in the collective regulation of foraging by harvester ants. Behavioral Ecology 22: 429-435. 
Gordon, D. M., Holmes, S. \& Nacu, S. 2007. The short-term regulation of foraging in harvester ants. Behavioral Ecology 19: 217-222.

Gordon, D. M., \& Mehdiabadi, N. J. (1999). Encounter rate and task allocation in harvester ants. Behavioral Ecology and Sociobiology, 45(5), 370-377. 2017. The neuropeptide corazonin controls social behavior and caste identity in ants. Cell 170: 748-759.e12. Behavioral Ecology 18: 451-455.

Greenwood, A. K., Ardekani, R., McCann, S. R., Dubin, M. E., Sullivan, A., Bensussen, S., Tavaré, S. \& Peichel, C. L. 2015. Genetic mapping of natural variation in schooling tendency in the threespine stickleback. G3: Genes|Genomes|Genetics 5: 761-769.

611 Hadfield, J. D. 2010. MCMC methods for multi-response generalized linear mixed models: The MCMCglmm R package. 2010 33: 22.

613 Hansen, C. \& Spuhler, K. 1984. Development of the National Institutes of Health genetically heterogeneous rat stock. Alcohol Clin Exp Res 8: 477-9.

615 Harris, J. W. \& Harbo, J. R. 1999. Heritability in honey bees (Hymenoptera: Apidae) of 616 characteristics associated with resistance to Varroa jacobsoni (Mesostigmata: Varroidae). 
Hedrick, P. W. \& Parker, J. D. 1997. Evolutionary genetics and genetic variation of haplodiploids and X-linked genes. Annual Review of Ecology and Systematics 28: 55-83.

Hunt, G. J., Amdam, G. V., Schlipalius, D., Emore, C., Sardesai, N., Williams, C. E., Rueppell, O., Guzmán-Novoa, E., Arechavaleta-Velasco, M., Chandra, S., Fondrk, M. K., Beye, M. \& Page, R. E. 2007. Behavioral genomics of honeybee foraging and nest defense. Naturwissenschaften 94: 247-267.

Hunt, G. J., Guzmán-Novoa, E., Fondrk, M. K. \& Page, R. E., Jr. 1998. Quantitative trait loci for

Ingram, K. K., Oefner, P. \& Gordon, D. M. 2005. Task-specific expression of the foraging gene in harvester ants. Molecular Ecology 14: 813-818.

Ingram Krista, K., Gordon Deborah, M., Friedman Daniel, A., Greene, M., Kahler, J. \& Peteru, S. 2016. Context-dependent expression of the foraging gene in field colonies of ants: the interacting roles of age, environment and task. Proceedings of the Royal Society B:

Jackson, D. E., \& Châline, N. (2007). Modulation of pheromone trail strength with food quality in Pharaoh's ant, Monomorium pharaonis. Animal Behaviour, 74(3), 463-470.

634 Jandt, J. M., Bengston, S., Pinter-Wollman, N., Pruitt, J. N., Raine, N. E., Dornhaus, A. \& Sih, A. 2014. Behavioural syndromes and social insects: personality at multiple levels.

637 Janzen, F. J. \& Stern, H. S. 1998. Logistic regression for empirical studies of multivariate selection. Evolution 52: 1564-1571. 
Jolles, J. W., Boogert, N. J., Sridhar, V. H., Couzin, I. D. \& Manica, A. 2017. Consistent individual differences drive collective behavior and group functioning of schooling fish. Current Biology 27: 2862-2868.e7.

King, E. G., Merkes, C. M., McNeil, C. L., Hoofer, S. R., Sen, S., Broman, K. W., Long, A. D. \& Macdonald, S. J. 2012. Genetic dissection of a model complex trait using the Drosophila Synthetic Population Resource. Genome Res 22: 1558-66.

Kingsolver, J. G., Hoekstra, H. E., Hoekstra, J. M., Berrigan, D., Vignieri, S. N., Hill, C. E., Hoang, A., Gibert, P. \& Beerli, P. 2001. The strength of phenotypic selection in natural populations. The American Naturalist 157: 245-261.

Kleineidam, C. J., Heeb, E. L., \& Neupert, S. (2017). Social interactions promote adaptive resource defense in ants. PLOS ONE, 12(9), e0183872.

Kocher, S. D., Mallarino, R., Rubin, B. E. R., Yu, D. W., Hoekstra, H. E. \& Pierce, N. E. 2018. The genetic basis of a social polymorphism in halictid bees. Nature Communications 9: 4338.

Krieger, M. J. 2005. To b or not to b: a pheromone-binding protein regulates colony social organization in fire ants. Bioessays 27: 91-9.

Kronauer, D. J. C. \& Libbrecht, R. 2018. Back to the roots: the importance of using simple insect societies to understand the molecular basis of complex social life. Current Opinion in Insect Science 28: 33-39.

Kruuk, L. E. B. 2004. Estimating genetic parameters in natural populations using the "animal model". Philosophical transactions of the Royal Society of London. Series B, Biological sciences 359: 873-890. 
661 Lande, R. \& Arnold, S. J. 1983. The measurement of selection on correlated characters. Evolution 37: 1210-1226.

Linksvayer, T. A. 2006. Direct, maternal, and sibsocial genetic effects on individual and colony traits in an ant. Evolution 60: 2552-2561.

Linksvayer, T. A. 2008. Queen-worker-brood coadaptation rather than conflict may drive colony resource allocation in the ant Temnothorax curvispinosus. Behavioral Ecology and Sociobiology, 62(5), 647-657. doi: 10.1007/s00265-007-0489-9

672 Linksvayer, T. A., Fondrk, M. K. \& Page Jr, R. E. 2009. Honeybee social regulatory networks are shaped by colony-level selection. The American Naturalist 173: E99-E107.

674 Löfqvist, J. (1976). Formic acid and saturated hydrocarbons as alarm pheromones for the ant Formica rufa. Journal of Insect Physiology, 22(10), 1331-1346.

676 Lucas, C. \& Sokolowski, M. B. 2009. Molecular basis for changes in behavioral state in ant social behaviors. Proceedings of the National Academy of Sciences 106: 6351-6356.

678 Lynch, M. \& Walsh, B. 1998. Genetics and analysis of quantitative traits. Sinauer.

679 McGlothlin, J. W., Moore, A. J., Wolf, J. B. \& Brodie III, E. D. 2010. Interacting phenotypes and the evolutionary process. III. Social evolution. Evolution 64: 2558-2574.

681 Mehdiabadi, N. J., Reeve, H. K. \& Mueller, U. G. 2003. Queens versus workers: sex-ratio 
Milne, C. P., Jr. 1985. Estimates of the heritabilities of and genetic correlation between two components of honey bee (Hymenoptera: Apidae) hygienic behavior: uncapping and removing. Annals of the Entomological Society of America 78: 841-844.

Modlmeier, A. P., Liebmann, J. E. \& Foitzik, S. 2012. Diverse societies are more productive: a lesson from ants. Proceedings of the Royal Society B: Biological Sciences 279: 21422150.

Möglich, M. 1978. Social organization of nest emigration in Leptothorax (Hym., Form.). Insectes Sociaux 25: 205-225.

Moore, A. J. 1990. The inheritance of social dominance, mating behaviour and attractiveness to mates in male Nauphoeta cinerea. Animal Behaviour 39: 388-397.

Moore, A. J., Brodie Iii, E. D. \& Wolf, J. B. 1997. Interacting phenotypes and the evolutionary process: I. Direct and indirect genetic effects of social interactions. Evolution 51: 13521362.

Moritz, R. F. A. 1989. Colony Level and within colony level selection in honeybees: A two allele population model for Apis mellifera capensis. Behavioral Ecology and bee workers (Apis mellifera L.). Behavioral Ecology and Sociobiology 21: 53-57.

701 Moritz, R. F. A., Southwick, E. E. \& Harbo, J. B. 1987. Genetic analysis of defensive behavior of honeybee colonies (Apis mellifera L.) in a field test. Apidologie 18: 27-42.

703 Morrissey, M. B. \& Sakrejda, K. 2013. Unification of regression-based methods for the analysis of natural selection. Evolution 67: 2094-2100. 
Mott, R., Talbot, C. J., Turri, M. G., Collins, A. C. \& Flint, J. 2000. A method for fine mapping quantitative trait loci in outbred animal stocks. Proceedings of the National Academy of Sciences 97: 12649-12654.

Mueller, L. D. (1997). Theoretical and empirical examination of density-dependent selection. Annual Review of Ecology and Systematics, 28(1), 269-288.

Muir, W. M. 2005. Incorporation of competitive effects in forest tree or animal breeding programs. Genetics 170: 1247-59.

Owen, R. E. 1986. Colony-level selection in the social insects: Single locus additive and nonadditive models. Theoretical Population Biology 29: 198-234.

714 Page, H., Sweeney, A., Pilko, A. \& Pinter-Wollman, N. 2018. Underlying mechanisms and ecological context of variation in exploratory behavior of the Argentine ant, Linepithema

Pinter-Wollman, N., Bala, A., Merrell, A., Queirolo, J., Stumpe, M. C., Holmes, S., \& Gordon, D. M. (2013). Harvester ants use interactions to regulate forager activation and

Pirchner, F. \& Bienefeld, K. 1991. Genetic correlations among several colony characters in the honey bee (Hymenoptera: Apidae) taking queen and worker effects into account. Annals of the Entomological Society of America 84: 324-331.

723 Planas-Sitjà, I., Deneubourg, J.-L., Gibon, C. \& Sempo, G. 2015. Group personality during collective decision-making: a multi-level approach. Proceedings of the Royal Society B: Biological Sciences 282: 20142515. 
Pontieri, L., Schmidt, A. M., Singh, R., Pedersen, J. S. \& Linksvayer, T. A. 2017. Artificial selection on ant female caste ratio uncovers a link between female-biased sex ratios and infection by Wolbachia endosymbionts. Journal of Evolutionary Biology 30: 225-234.

Pratt, S. C. (2017) Nest site choice in social insects. Encyclopedia of Animal Behavior. 2: 534 540.

R Core Team. 2014. R: a language and environment for statistical computing. $\mathrm{R}$

Ratnieks, F. L. W. \& Reeve, H. K. 1992. Conflict in single-queen hymenopteran societies: the structure of conflict and processes that reduce conflict in advanced eusocial species. Journal of Theoretical Biology 158: 33-65.

Reuter, M. \& Keller, L. 2001. Sex ratio conflict and worker production in eusocial Hymenoptera. The American Naturalist 158: 166-177.

Rinderer, T. E., Collins, A. M. \& Brown, M. A. 1983. Heritabilities and correlations of the honey bee: response to Nosema apis, longevity, and alarm response to isopentyl acetate. Apidologie 14: 79-85.

741 Robinson, E. J. H., Jackson, D. E., Holcombe, M. \& Ratnieks, F. L. W. 2005. 'No entry’ signal in ant foraging. Nature 438: 442.

743 Schmidt, A. M. (2010). The invasion biology and sociogenetics of pharaoh ants. Museum Tusculanum.

745 Schmidt, A. M., d'Ettorre, P. \& Pedersen, J. S. 2010. Low levels of nestmate discrimination $746 \quad$ despite high genetic differentiation in the invasive pharaoh ant. Frontiers in Zoology 7: 
Sih, A., Bell, A. \& Johnson, J. C. 2004. Behavioral syndromes: an ecological and evolutionary overview. Trends in Ecology \& Evolution 19: 372-378.

Simons, A. M. \& Roff, D. A. 1994. The effect of environmental variability on the heritabilities of traits of a field cricket. Evolution 48: 1637-1649.

Smith, B. R. \& Blumstein, D. T. 2008. Fitness consequences of personality: a meta-analysis. Behavioral Ecology 19: 448-455.

754 Spivak, M. 1996. Honey bee hygienic behavior and defense against Varroa jacobsoni. Apidologie 27: 245-260.

Sumpter, D. J. T. \& Beekman, M. 2003. From nonlinearity to optimality: pheromone trail foraging by ants. Animal Behaviour 66: 273-280.

Tang, W., Zhang, G., Serluca, F., Li, J., Xiong, X., Coble, M., Tsai, T., Li, Z., Molind, G., Zhu, P. \& Fishman, M. C. 2018. Genetic architecture of collective behaviors in zebrafish.

Tarpy, D. R., Gilley, D. C. \& Seeley, T. D. 2004. Levels of selection in a social insect: A review of conflict and cooperation during honey bee (Apis mellifera) queen replacement.

764 Trivers, R. L. \& Hare, H. 1976. Haploidploidy and the evolution of the social insect. Science 191: 249-63.

Tsuji, K. 1994. Inter-colonial selection for the maintenance of cooperative breeding in the ant 35: 109-113. 
Tsuji, K. 1995. Reproductive Conflicts and Levels of Selection in the Ant Pristomyrmex pungens: Contextual Analysis and Partitioning of Covariance. The American Naturalist 146: 586-607.

Valdar, W., Solberg, L. C., Gauguier, D., Burnett, S., Klenerman, P., Cookson, W. O., Taylor, complex traits in heterogeneous stock mice. Nat Genet 38: 879-87.

van Oers, K., de Jong, G., van Noordwijk, A. J., Kempenaers, B. \& Drent, P. J. 2005. Behaviour 142: 1185-1206.

Wade, M. J. 1976. Group selections among laboratory populations of Tribolium. Proc Natl Acad Sci U S A 73: 4604-7.

Wade, M. J., Bijma, P., Ellen, E. D. \& Muir, W. 2010. Group selection and social evolution in domesticated animals. Evolutionary applications 3: 453-465.

782 Walsh, J. T., Warner, M. R., Kase, A., Cushing, B. J. \& Linksvayer, T. A. 2018. Ant nurse workers exhibit behavioural and transcriptomic signatures of specialization on larval stage. Animal Behaviour 141: 161-169.

785 Wang, J., Ross, K. G. \& Keller, L. 2008. Genome-wide expression patterns and the genetic architecture of a fundamental social trait. PLOS Genetics 4: e1000127.

787 Wang, J., Wurm, Y., Nipitwattanaphon, M., Riba-Grognuz, O., Huang, Y.-C., Shoemaker, D. \& Keller, L. 2013. A Y-like social chromosome causes alternative colony organization in fire ants. Nature 493: 664. 
Wark, A. R., Greenwood, A. K., Taylor, E. M., Yoshida, K. \& Peichel, C. L. 2011. Heritable differences in schooling behavior among threespine stickleback populations revealed by a novel assay. PLOS ONE 6: e18316.

Warner, M. R., Lipponen, J. \& Linksvayer, T. A. 2018. Pharaoh ant colonies dynamically regulate reproductive allocation based on colony demography. Behavioral Ecology and Sociobiology 72: 31 .

Warner, M. R., Mikheyev, A. S. \& Linksvayer, T. A. 2017. Genomic signature of kin selection in an ant with obligately sterile workers. Molecular Biology and Evolution 34: 17801787.

Wetterer, J. 2010. Worldwide spread of the pharaoh ant, Monomorium pharaonis (Hymenoptera: Formicidae).

Wilson, A. J., Reale, D., Clements, M. N., Morrissey, M. M., Postma, E., Walling, C. A., Kruuk, L. E. \& Nussey, D. H. 2010. An ecologist's guide to the animal model. J Anim Ecol 79: $13-26$. 2019. Collective personalities: present knowledge and new frontiers. Behavioral Ecology and Sociobiology 73: 31. 
811 Table 1. Linear and quadratic selection estimates for behaviors using either reproductive

812 (R) or worker $(W)$ production as the measurement of fitness.

Trait

Estimate SE

p Estimate

SE

(W)

\begin{tabular}{|c|c|c|c|c|c|c|}
\hline \multicolumn{4}{|l|}{ Linear } & \multicolumn{3}{|c|}{ T } \\
\hline Foraging & 0.245 & 0.071 & $<0.001$ & 0.122 & 0.047 & 0.008 \\
\hline Aggression & -0.054 & 0.070 & 0.446 & 0.007 & 0.048 & 0.916 \\
\hline Exploratory rate & -0.088 & 0.052 & 0.048 & -0.022 & 0.041 & 0.586 \\
\hline Group exploration & -0.033 & 0.051 & 0.498 & 0.027 & 0.047 & 0.788 \\
\hline Colony exploration & -0.014 & 0.063 & 0.810 & 0.015 & 0.049 & 0.788 \\
\hline \multicolumn{7}{|l|}{ Quadratic } \\
\hline Foraging & 0.025 & 0.059 & 0.576 & 0.019 & 0.022 & 0.130 \\
\hline Aggression & 0.003 & 0.010 & 0.248 & 0.00005 & 0.049 & 0.412 \\
\hline Exploratory rate & 0.008 & 0.010 & 0.106 & 0.050 & 0.051 & 0.206 \\
\hline Group exploration & 0.001 & 0.005 & 0.258 & 0.085 & 0.078 & 0.116 \\
\hline Colony exploration & 0.0002 & 0.006 & 0.306 & 0.0002 & 0.004 & 0.266 \\
\hline
\end{tabular}




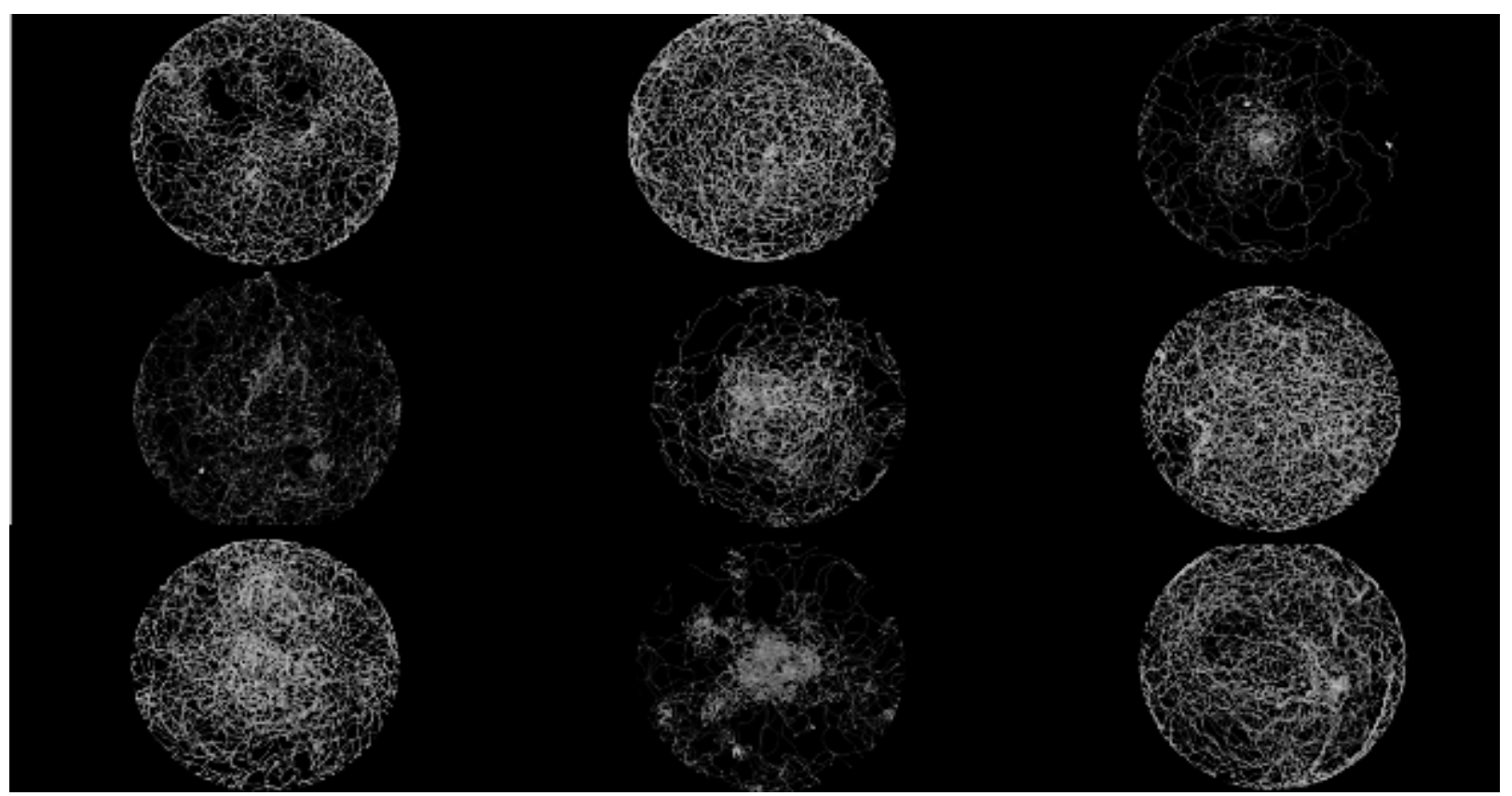

816 Figure 1. Nine representative plots showing variation among colony genotypes in the

817 exploratory patterns of groups of five foragers. The plots show the tracks (white pixels) of the

818 ants as they explore a novel arena. 


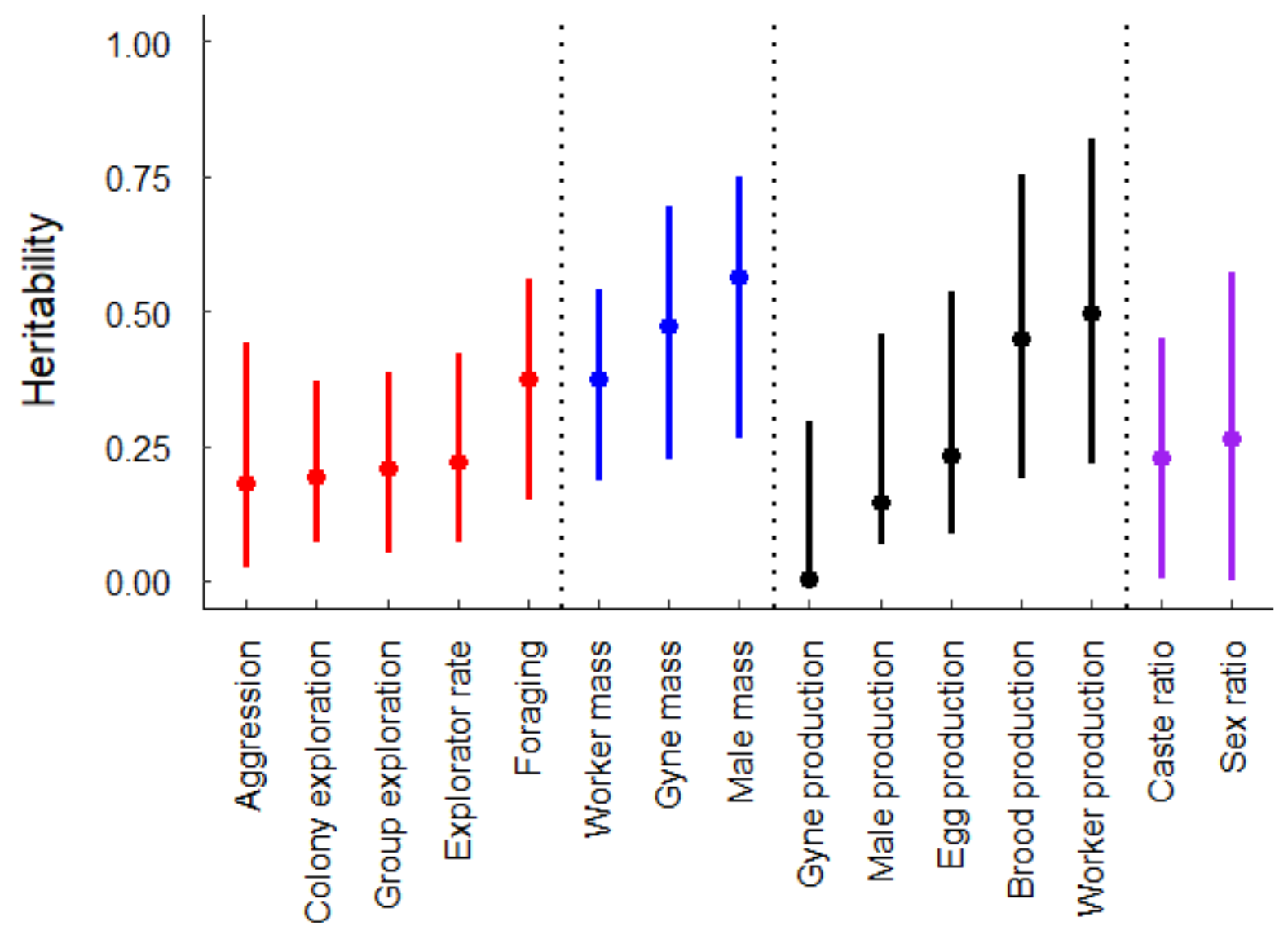

821 Figure 2. Caterpillar plot of heritability estimates $+/-95 \%$ confidence intervals grouped by

822 category. Collective behaviors (red), body mass (blue), colony productivity (black), and caste 823 and sex ratio (purple) are designated by different colors. 


\section{A. Phenotypic correlations}

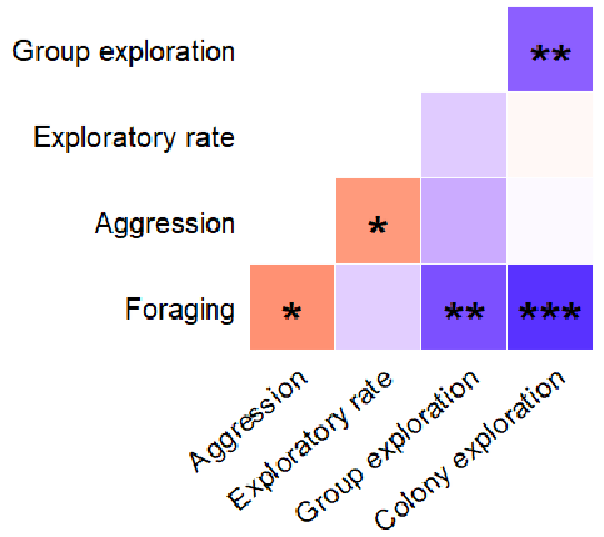

\section{B. Genetic correlations}

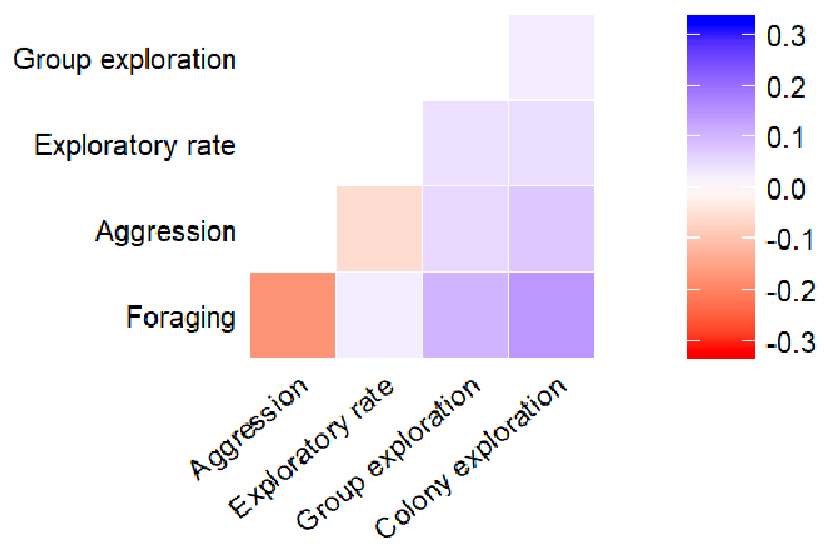

826 Figure 3. Heatmaps showing phenotypic (A) and genetic (B) correlations between collective

827 behaviors. For the phenotypic correlations, asterisks within cells correspond to p values (adjusted

828 for multiple comparisons; $\mathrm{p}<0.05=* ; \mathrm{p}<0.01=* * ; \mathrm{p}<0.001=* * * ;$ no symbol indicates $\mathrm{p}>$

829 0.05) and the colors correspond to the magnitude and sign of the Spearman rank correlation

830 coefficient. None of the genetic correlations were significant (all 95\% CIs overlapped with zero). 


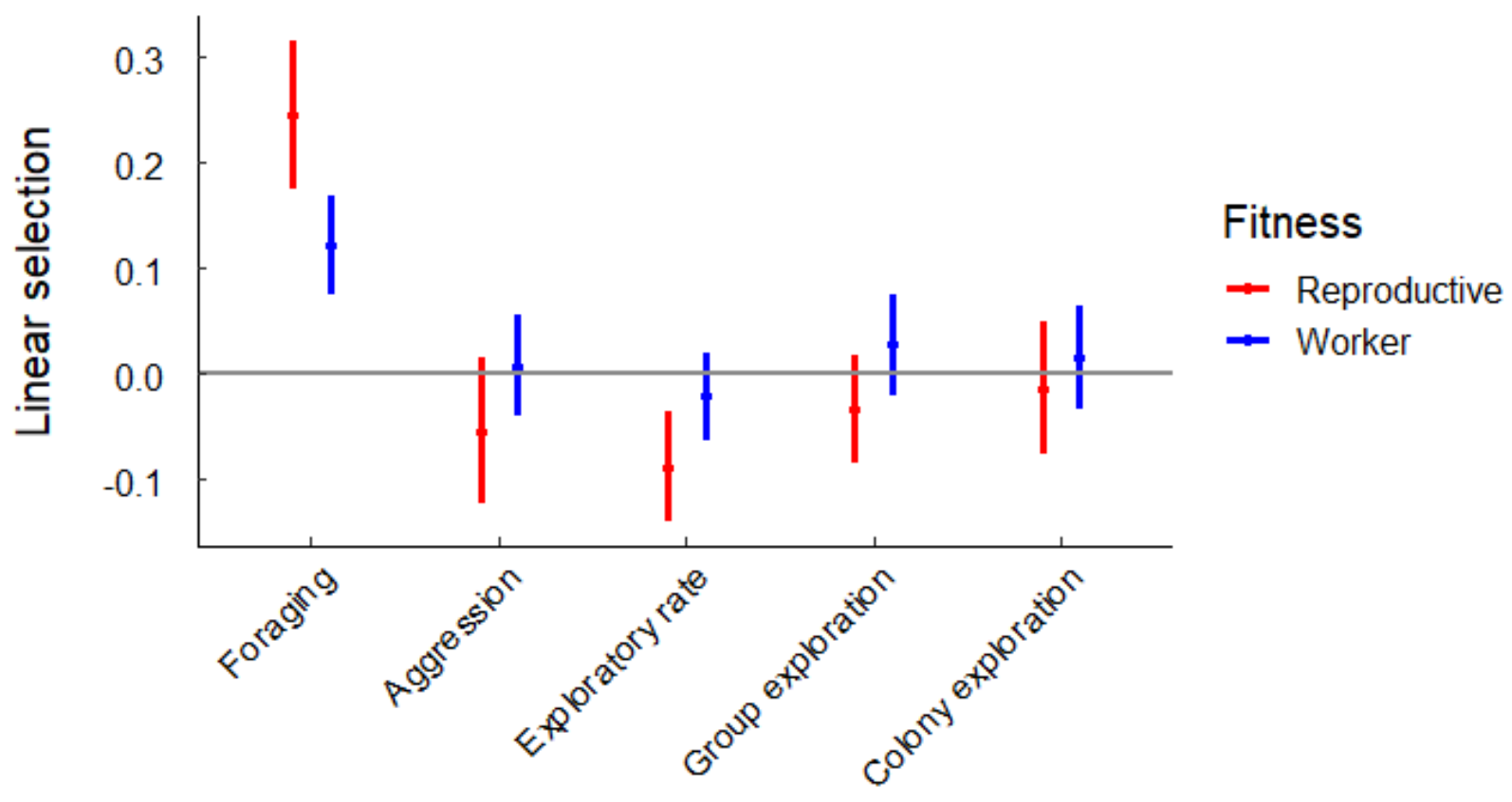

834 Figure 4. Caterpillar plot showing linear (a) and quadratic (b) selection gradients +/- SE.

835 Asterisks indicate estimates that are significant. Positive values indicate directional selection for 836 increased trait values (e.g., there is directional selection for increased foraging rate when fitness

837 is measured by the number of reproductives or workers produced) and negative values indicate

838 directional selection for decreased trait values (e.g., there is directional selection for decreased

839 exploratory rate when fitness is measured as the number of reproductives produced). 